\title{
Explorando relações entre o comportamento social e o desempenho acadêmico em crianças $^{1}$
}

\section{Exploring the social behaviour and academic performance relations in children}

Fabio Biasotto Feitosa*

Universidade Federal de Rondônia - UNIR, Porto Velho, RO, Brasil

Zilda A. P. Del Prette**

Universidade Federal de São Carlos - UFSCAR, São Carlos, SP, Brasil

\author{
Almir Del Prette*** \\ Universidade Federal de São Carlos - UFSCAR, São Carlos, SP, Brasil
}

Sonia Regina Loureiro****

Universidade de São Paulo - USP, Ribeirão Preto, SP, Brasil

\begin{abstract}
RESUMO
A frequente correlação entre o comportamento social de crianças e o desempenho acadêmico tem sido amplamente reconhecida. Objetiva-se investigar as possíveis correlações entre habilidades sociais, problemas de comportamento e desempenho acadêmico de crianças. A amostra consistiu de 80 crianças de escolas públicas da região sudeste $(60 \%)$ e norte $(40 \%)$ do Brasil, com idade média de 8,15 anos e desvio padrão igual a 0,68 , sendo $57,5 \%$ meninos e $42,5 \%$ meninas. As crianças foram avaliadas por meio do Teste de Desempenho Escolar e os pais e professoras dessas crianças responderam, de forma independente, ao Sistema de Avaliação de Habilidades Sociais. As variáveis foram tratadas pelo teste de correlação de Spearman. Os resultados mostraram que as habilidades sociais têm maior probabilidade de promover o desempenho escolar de crianças do que os problemas de comportamento de prejudicá-lo, com implicações estratégicas para a intervenção e prevenção das dificuldades de aprendizagem.
\end{abstract}

Palavras-chave: Habilidades sociais, Problemas de comportamento, Desempenho acadêmico.

\begin{abstract}
The relationship between social behavior and academic performance in children has been widely recognized. The present study aims to investigate possible correlations between social skills, behavioural problems and academic performance in children. The sample consisted of 80 children attending public schools in the southeast $(60 \%)$ and north $(40 \%)$ of Brazil, with a mean age of 8.15 years (standard deviation equal to 0.68 ), 57.5\% boys and $42.5 \%$ girls. The children completed the School Achievement Test and the parents and teachers, independently, completed the Social Skills Rating System. The variables were treated by the Spearman correlation test. The results showed that the likelihood of social skills contributing to
\end{abstract}


Fabio Biasotto Feitosa, Zilda A. P. Del Prette, Almir Del Prette, Sonia Regina

children's school achievement was higher than the eventual damages caused by behavioral problems in this performance, what has strategic implications for the intervention and prevention of learning disabilities.

Keywords: Social skills, Behaviour problems, Academic performance.

O estudo do comportamento social pode ser feito sob diferentes enfoques teóricos, no presente estudo tal temática será abordada sob a perspectiva das habilidades sociais. O campo das habilidades sociais inclui conceitos e procedimentos de avaliação-intervenção desenvolvidos a partir de estudos na área da psicologia cognitivocomportamental, teoria da aprendizagem social, abordagem sistêmica e ecológica, entre outras, abordando temáticas diversas tais como os recursos para o desenvolvimento de comportamentos necessários ao ajustamento social e escolar, a saúde e a qualidade de vida. De forma breve, apresentar-se-á alguns aspectos conceituais relativos às habilidades sociais e suas implicações para o comportamento social, o desempenho acadêmico e a adaptação da criança na escola.

\section{Habilidades sociais}

No campo das habilidades sociais considera-se três conceitos chaves que articuladamente embasam os estudos e as intervenções com o foco nas relações interpessoais: desempenho social, habilidades sociais e competência social. De acordo com Del Prette e Del Prette (2001, p.31),

o desempenho social refere-se à emissão de um comportamento ou seqüência de comportamentos em uma situação social qualquer [...], o termo habilidades sociais aplica-se à noção de existência de diferentes classes de comportamentos sociais no repertório do indivíduo para lidar de maneira adequada com as demandas das situações sociais [e] o conceito de competência social tem sentido avaliativo que remete aos efeitos do desempenho das habilidades nas situações vividas pelo indivíduo.

Dentre as principais hipóteses explicativas para as dificuldades interpessoais estão (DEL PRETTE; DEL PRETTE, 2005): o déficit de aquisição (ausência total de habilidade requerida), déficit de desempenho (presença ocasional de habilidade requerida) e o déficit de fluência (habilidade emitida sem a proficiência requerida para produzir os resultados esperados), inibição mediada pela ansiedade (esquiva e fuga de situações), inibição cognitivamente mediada (problemas de crenças, expectativas, padrões negativistas ou perfeccionistas de pensamento), problemas de percepção social (ausência ou alterações na decodificação de sinais sociais) e problemas de processamento de estímulos do ambiente (demora na discriminação de símbolos, déficit de atenção, decodificação de sinais 
sociais alterada por estereótipos, falha na identificação de alternativas).

As habilidades sociais podem ser classificadas, ainda, em alguns conjuntos, como (DEL PRETTE; DEL PRETTE, 2001): habilidades sociais de comunicação (exemplo: iniciar, manter e encerrar conversação), habilidades sociais de civilidade (exemplo: apresentarse), habilidades sociais assertivas (exemplo: manifestar opinião), habilidades sociais empáticas (exemplo: expressar apoio), entre outras. A amplitude de manifestações comportamentais das habilidades sociais coloca em destaque o seu papel central nas interações e comportamentos sociais como reconhecidos indicadores de adaptação.

\section{Habilidades sociais e adaptação na escola}

A importância da qualidade das relações interpessoais sobre 0 desenvolvimento e a aprendizagem na infância tem sido amplamente reconhecida (DEL PRETTE; DEL PRETTE, 2001; DEL PRETTE; DEL PRETTE, 2005; KAZDIN; WEISZ, 2004; NOVAK; PELÁEZ, 2003). A correlação significativa entre déficits de habilidades sociais, problemas de comportamento e dificuldades de aprendizagem (BANDEIRA; COL., 2006; DIPERNA, 2006; RIMM-KAUFMAN; CHIU, 2007; RUTHERFORD; DUPAUL; JITENDRA, 2008) sugere que 0 ajustamento escolar de crianças envolve, em um sentido amplo, tanto aspectos acadêmicos como interpessoais.

Ainda que essa associação entre baixa competência social e dificuldades de aprendizagem esteja bem documentada na literatura, alguns autores (BANDEIRA ET AL., 2006; DIPERNA, 2006; KAVALE; FORNESS, 1996; SWANSON; MALONE, 1992) destacam que a natureza dessa relação, suas características e mecanismos ainda carecem de maior investigação.

Ao analisar a literatura, verifica-se a emergência de uma hipótese explicativa para esta relação, ou seja, as habilidades sociais podem ter uma função instrumental ou facilitadora da aprendizagem acadêmica (DIPERNA, 2006; FAD, 1990; WENTZEL, 1991). Como os processos de ensino-aprendizagem ocorrem basicamente em ambiente social, sob interações sociais educativas da criança com o educador e com seus pares (DEL PRETTE; DEL PRETTE, 2003; DEL PRETTE; DEL PRETTE, 2005; ZINS; WALBERG; WEISSBERG, 2004), considera-se razoável supor que a qualidade das relações interpessoais estabelecidas nesse contexto podem afetar positiva ou negativamente o desempenho acadêmico. Na literatura internacional recente, evidências recorrentes vêm apoiando essa hipótese (CAPRARA; BARBARANELLI; PASTORELLI; BANDURA; ZIMBARDO, 2000; DIPERNA, 2006; EISENHOWER; BAKER; BLACHER, 2007; MCCLELLAND; ACOCK; MORRISON, 2006). 
No Brasil, evidências empíricas nessa direção também foram encontradas. Em estudo de regressão logística (DEL PRETTE; DEL PRETTE; OLIVEIRA; GRESHAM; VANCE, s.d.), indicadores de habilidades sociais de cooperação e sociabilidade foram preditores confiáveis do pertencimento da criança a grupo com dificuldade de aprendizagem em relação ao grupo sem dificuldade de aprendizagem, evidenciando a importância dessas habilidades no rendimento acadêmico. Em estudo experimental com 16 estudantes que apresentavam dificuldades de leitura e escrita (seis meninas e dez meninos entre sete e treze anos), Molina e Del Prette (2006) encontraram que o grupo submetido a programa de habilidades sociais apresentou as melhoras esperadas no repertório social e, adicionalmente, ganhos acadêmicos, enquanto que o grupo submetido a treinamento acadêmico melhorou o desempenho acadêmico mas não obteve ganhos no repertório social. As autoras concluem que os resultados favorecem a hipótese de relação funcional entre habilidades sociais e o desempenho acadêmico.

Outro ponto a ser destacado diz respeito à descrição do perfil das crianças encaminhadas para atendimento clínico por dificuldades de aprendizagem. Constata-se que essas geralmente são apresentadas com uma série de problemas comportamentais, tais como desobediência, irritabilidade, impaciência, agitação, ansiedade, oposição, esquiva, insegurança, timidez, dispersão, isolamento, preocupação, brigas e destruição de objetos (MARTURANO; PARREIRA; BENZONI, 1997; ROMARO; CAPITÃO, 2003). As crianças com dificuldades de aprendizagem usariam estratégias para a solução de problemas interpessoais menos aceitáveis socialmente, assumindo um padrão polarizado como ganhar ou perder, caracterizando pouca habilidade de negociação com colegas (CARLSON, 1987). Nesse sentido, os problemas de comportamento poderiam competir com a atenção às aulas ou mesmo com as interações sociais mais habilidosas com professores e colegas, prejudicando o desempenho acadêmico.

A associação de desempenho acadêmico aos comportamentos sociais foi também verificada no estudo longitudinal de Caprara e cols. (2000), constatando, com 294 crianças italianas de 8,5 anos de idade, que após cinco anos o efeito positivo sobre o desempenho acadêmico e o status sociométrico dos comportamentos prossociais (oferecer ajuda, compartilhar, ser gentil e cooperar) foi mais robusto, comparativamente ao efeito negativo de comportamentos antissociais (como a agressividade).

Da análise das possíveis relações entre desempenho acadêmico e comportamento social de crianças escolares, abordadas nesses estudos, emerge a questão da pesquisa aqui abordada. Pretende-se verificar, em uma amostra brasileira, o peso das habilidades sociais, comparativamente aos problemas de comportamento, em sua 
associação com o desempenho acadêmico de crianças. Considera-se que respostas a essa questão poderão instrumentar o planejamento de programas comportamentais voltados a crianças em processo de escolarização, além de favorecer estratégias de intervenção e prevenção das dificuldades de aprendizagem. Diante disso, objetivouse verificar as possíveis correlações entre habilidades sociais, problemas de comportamento e desempenho acadêmico de crianças.

\section{Método}

O presente estudo foi conduzido sob um delineamento ex-post-facto do tipo correlacional. Segundo Gil (1999), esse delineamento tem certa semelhança com o experimental, pois ambos buscam uma investigação sistemática e empírica de relações funcionais, mas difere deste na ausência de controle direto sobre as variáveis independentes, uma vez que suas manifestações já ocorreram. As inferências sobre a relação entre variáveis são feitas sem observação direta, a partir de indicadores de variação concomitante entre as variáveis de interesse para posterior discussão com base na literatura especializada. Os dados, objeto de análise deste estudo, são baseados na amostra total da pesquisa de doutorado de Feitosa (2007).

\section{Participantes}

A amostra consistiu de 80 crianças de escolas públicas da região sudeste $(60 \%)$ e norte $(40 \%)$ do Brasil, com idade média de 8,15 anos e desvio padrão igual a 0,68 , sendo $57,5 \%$ meninos e $42,5 \%$ meninas. Conforme avaliação pelo Critério Brasil ${ }^{2}$, a maioria com nível socioeconômico baixo $(D=38,8 \%$ e $C=38,8 \%)$ e uma proporção pequena de nível socioeconômico médio e alto $(B 2=15 \%, B 1=3,8 \%$ e $\mathrm{A} 2=3,8 \%)$. Quanto à escolaridade, as crianças frequentavam a segunda $(73,8 \%)$ e terceira $(26,2 \%)$ séries do ensino fundamental. Participaram, como informantes, os pais ou responsáveis e as sete professoras dessas crianças. Verificaram-se algumas omissões nas avaliações de algumas variáveis pelos professores e familiares por falha no preenchimento de alguns itens, sendo considerado missing, o que faz com que a amostra apareça reduzida a até 72 crianças em algumas análises.

Os dois grupos de crianças de regiões brasileiras distintas foram unificados para as análises estatísticas considerando a semelhança entre eles. Conforme mostra a Tabela 1, pelo teste Mann-Whitney U, as únicas diferenças significativas entre os grupos foram em relação aos problemas de comportamento na avaliação dos pais da região norte (ligeiramente menos frequente) e no nível socioeconômico, que ficou maior na amostra do sudeste (sudeste=C, norte=D), sem prejuízo para as análises do presente estudo. 
Fabio Biasotto Feitosa, Zilda A. P. Del Prette, Almir Del Prette, Sonia Regina Loureiro Explorando relações

Tabela 1. Médias das variáveis em estudo por região.

\begin{tabular}{|c|c|c|c|c|c|}
\hline Variáveis & $\begin{array}{c}\text { Cidade da } \\
\text { coleta }\end{array}$ & $N$ & Média & $\begin{array}{l}\text { Desvio } \\
\text { Padrão }\end{array}$ & Z \\
\hline \multirow[t]{3}{*}{ Habilidades Sociais $^{a}$} & São Paulo-SP & 48 & 31,79 & 8,86 & \multirow{2}{*}{$-1,35$} \\
\hline & Cacoal-Ro & 32 & 34,75 & 15,01 & \\
\hline & Total & 80 & & & \\
\hline \multirow{3}{*}{$\begin{array}{l}\text { Problemas de } \\
\text { Comportamento }\end{array}$} & São Paulo-SP & 48 & 9,60 & 7,74 & \multirow{2}{*}{$-1,65$} \\
\hline & Cacoal-RO & 26 & 12,96 & 8,87 & \\
\hline & Total & 74 & & & \\
\hline \multirow[t]{3}{*}{ Habilidades Sociais $^{b}$} & São Paulo-SP & 47 & 45,83 & 9,88 & \multirow{2}{*}{$-1,42$} \\
\hline & Cacoal-RO & 31 & 49,48 & 11,83 & \\
\hline & Total & 78 & & & \\
\hline \multirow{3}{*}{$\begin{array}{l}\text { Problemas de } \\
\text { Comportamento }\end{array}$} & São Paulo-SP & 46 & 13,89 & 5,11 & \multirow{2}{*}{$-2,24^{*}$} \\
\hline & Cacoal-RO & 26 & 10,85 & 6,00 & \\
\hline & Total & 72 & & & \\
\hline \multirow[t]{3}{*}{ Nivel Socioeconômico } & São Paulo-SP & 48 & 3,23 & 1,15 & \multirow{2}{*}{$-2,79 * *$} \\
\hline & Cacoal-Ro & 32 & 2,53 & 0,57 & \\
\hline & Total & 80 & & & \\
\hline \multirow{3}{*}{$\begin{array}{c}\text { Desempenho } \\
\text { Acadêmico }\end{array}$} & São Paulo-SP & 48 & 62,75 & 40,14 & \multirow{2}{*}{$-0,60$} \\
\hline & Cacoal-RO & 32 & 74,25 & 26,23 & \\
\hline & Total & 80 & & & \\
\hline
\end{tabular}

\section{I nstrumentos}

Sistema de Avaliação de Habilidades Sociais. Trata-se de instrumento validado para o Brasil (SSRS-BR, DEL PRETTE, Z., 2003; BANDEIRA; DEL PRETTE; DEL PRETTE; MAGALHÃES, 2009), a partir do Social Skills Rating System (SSRS, GRESHAM; ELLIOTT, 1990), usado para avaliar habilidades sociais e problemas de comportamento. $\mathrm{Na}$ amostra de validação, foi encontrado índice satisfatório de consistência interna e estabilidade temporal teste-reteste. Foram utilizadas as versões de pais (coeficiente de alpha entre 0,83 e 0,86) e professores (coeficiente de alfa entre 0,91 e 0,94) para a frequência com que a criança apresenta habilidades sociais (exemplo: cooperação) e problemas de comportamento (exemplo: hiperatividade) descritas no instrumento, assinalando suas respostas em uma escala de três pontos (nunca, às vezes, sempre). As versões para pais e professores são bastante semelhantes quanto as área avaliadas.

Teste de Desempenho Escolar. O Teste de Desempenho Escolar-TDE é um instrumento validado no Brasil (STEIN, 1994), de aplicação individual, para avaliação das habilidades de escrita, aritmética e leitura. O teste produz escores brutos de acertos em cada habilidade e no total geral, sendo esses valores convertidos em percentis classificados em inferior, médio ou superior. No presente estudo, utilizou-se apenas o escore bruto de cada criança no total geral. No estudo original psicométrico com o TDE foram referidos como 
Fabio Biasotto Feitosa, Zilda A. P. Del Prette, Almir Del Prette, Sonia Regina Loureiro

indicadores de confiabilidade os seguintes coeficientes alfa: Escrita $=0,95$, Aritmética $=0,93$, Leitura $=0,99$ e Total=0,99 (STEIN, 1994).

\section{Procedimento}

O projeto foi submetido ao Comitê de Ética e aprovado (CEP/UFSCar no. 134/2005). A aplicação dos instrumentos com os alunos foi realizada em sala com privacidade, de forma individual, pelo próprio pesquisador nas duas escolas participantes. Os professores receberam os nomes dos alunos, cujos pais consentiram a participação na pesquisa, e foram orientados a avaliarem cada criança nos itens do SSRS-BR. Os familiares avaliaram seus filhos e informaram os dados socioeconômicos, quando da participação em uma reunião regular realizada na escola. A participação ocorreu após assinarem o termo de consentimento com a pesquisa.

Os instrumentos foram codificados conforme as recomendações técnicas e tratados por procedimentos estatísticos utilizando-se para tal o programa estatístico SPSS for Windows 15.0 (2005) e do teste de correlação de Spearman, adotando-se o nível de significância de $p<0,05$.

\section{Resultados}

Apresentar-se-á, a seguir, as correlações entre as variáveis avaliadas por pais e professores, por meio do teste de correlação de Spearman, destacando os resultados significativos. $\mathrm{Na}$ Tabela 2 são apresentados os dados relativos às avaliações dos pais.

Tabela 2. Correlações entre variáveis tendo pais como avaliadores do desempenho social das crianças.

\begin{tabular}{|c|c|c|c|}
\hline \multirow{2}{*}{\multicolumn{4}{|c|}{$\frac{\text { Variável }}{\text { 1. Habilidades Sociais }}$}} \\
\hline & & & \\
\hline 2. Problemas de comportamento & $\begin{array}{c}-0,529 * * \\
(N=72)\end{array}$ & & \\
\hline 3. Desempenho Acadêmico & $\begin{array}{l}0,260 * \\
(N=78)\end{array}$ & $\begin{array}{r}-0,228 \\
(N=72)\end{array}$ & \\
\hline
\end{tabular}

Verifica-se, com significância estatística, correlação moderada, negativa, entre habilidades sociais e problemas de comportamento e correlação fraca, positiva, com o desempenho acadêmico. Na Tabela 3 são apresentadas as correlações entre as variáveis avaliadas pelos professores. 
Fabio Biasotto Feitosa, Zilda A. P. Del Prette, Almir Del Prette, Sonia Regina

Tabela 3. Correlações entre variáveis tendo professores como avaliadores do desempenho social das crianças.

\begin{tabular}{lccc}
\hline \multicolumn{1}{c}{ Variável } & 1 & 2 & 3 \\
\hline 1. Habilidades Sociais & & & \\
\hline 2. Problemas de Comportamento & $-0,474 *$ & \\
& $(N=74)$ & \\
\hline 3. Desempenho Acadêmico & $0,439 * *$ & 0,145 \\
& $(N=80)$ & $(N=74)$ \\
\hline
\end{tabular}

Observa-se segundo os professores, com significância estatística, correlação moderada, inversa, entre as habilidades sociais e os problemas de comportamento em crianças, e correlação moderada e positiva com o desempenho acadêmico. Destaca-se que os problemas de comportamento não se correlacionaram com o desempenho acadêmico segundo ambos informantes. Entretanto, o índice de correlação entre habilidades sociais e desempenho acadêmico foi maior na avaliação dos professores.

\section{Discussão}

Constatou-se, com base nas avaliações de pais e professores, a correlação entre habilidades sociais e problemas de comportamento e desempenho acadêmico registradas na literatura (BANDEIRA ET.AL., 2006; DIPERNA, 2006; RIMM-KAUFMAN; CHIU, 2007; RUTHERFORD; DUPAUL; JITENDRA, 2008). A ausência de correlação entre problemas de comportamento e desempenho acadêmico, reforçada pela dupla avaliação de pais e professores, é um achado que parece endossar a discussão quanto ao papel das habilidades como um recurso promotor do desempenho acadêmico.

Nesse sentido, os dados obtidos se assemelham aos de Caprara e cols. (2000) quanto à constatação de que a ausência de habilidades sociais pode ser mais prejudicial para o desempenho acadêmico das crianças do que os seus problemas de comportamento. Em outras palavras, as habilidades sociais têm maior probabilidade de contribuir para o desempenho escolar de crianças do que os problemas de comportamento para comprometê-lo. Os dados se assemelham também aos de Molina e Del Prette (2006) quanto ao recurso facilitador das habilidades sociais para o rendimento acadêmico de crianças. Sob tal perspectiva, os dados do presente estudo confirmam a noção de habilidades sociais como facilitadores do desempenho acadêmico das crianças, competindo, em relevância, com o prejuízo associado aos problemas de comportamento.

A referida perspectiva, confirmada neste presente estudo com crianças de uma amostra brasileira, composta por dois grupos geograficamente bastante separados, aponta para a pertinência da 
proposição de programas de habilidades sociais na assistência a crianças com problemas de comportamento e/ou dificuldades de aprendizagem, como propostos por Del Prette e Del Prette (2005) e Zins e cols. (2004), visando favorecer a inserção social de alunos na escola e na comunidade.

Propostas como essas são baseadas na concepção das escolas como espaços sociais, sendo o aprendizado escolar um processo social, de maneira que os alunos não aprendem sozinhos mas em colaboração com professores, na compainha dos seus pares e com o apoio da família (ZINS et. al., 2004). O enfrentamento de demandas interpessoais requer que as crianças desenvolvam um repertório cada vez mais elaborado de habilidades sociais, com consequente melhora da qualidade de vida, adaptação social e rendimento acadêmico (DEL PRETTE; DEL PRETTE, 2005).

A promoção das habilidades sociais nas escolas pode ser feita, por recursos diversos como por exemplo, mediante programas sistemáticos como os "3Cs" (Three Cs - Cooperation, Conflit Resolution, and Civic Values) de Johnson e Johnson (2001). O referido programa consiste no desenvolvimento de habilidades de cooperação, resolução de conflitos e valores cívicos na criança. A cooperação é promovida por meio de atividades em pequenos grupos com objetivos comuns que aumentam a interdependência entre os pares, a divisão de recursos e os papéis complementares, reduzindo o padrão usual competitivo e individualista da sala de aula. A resolução de conflitos é ensaiada em atividades que propiciem lidar com controvérsias acadêmicas, alcançando uma conclusão capaz de ser aceita pelos grupos antes divergentes. E os valores cívicos, resumidos na noção de bem comum, podem ser desenvolvidos mediante 0 desempenho de papéis voluntários.

Nessa direção, no Brasil, Del Prette e Del Prette (2005) propõem o uso de vivências para o trabalho com crianças, que incluem em seus objetivos a promoção de habilidades empáticas, assertivas, de civilidade entre outras. As vivências estruturam situações análogas às do cotidiano, possibilitando o ensaio de papéis e a resolução de problemas interpessoais, constituindo, assim, um importante recurso comportamental, lúdico e pedagógico para a promoção do desenvolvimento social e escolar das crianças.

Muitas escolas norte-americanas já incluíram no currículo regular programas educativos visando a promoção de habilidades sociais. Dentre outras agências educacionais envolvidas nesse processo de implementação de novos currículos, convém destacar o CASEL (Collaborative for the Advancement of Social and Emotional Learning), uma organização fundada em dezembro de 1994 pelo Departamento de Psicologia da Universidade de Illinois (E.U.A.), envolvendo educadores, cientistas, políticos e outros cidadãos preocupados em identificar modelos educacionais que maximizem o 
desenvolvimento social e emocional das crianças, seu desempenho acadêmico, habilidades pessoais e cidadania. Em uma análise de mais de uma década de estudos nessa temática, considera-se que os programas de habilidades sociais têm apresentado resultados eficazes.

Com base em um estudo de metanálise, CASEL (2008) mostrou que, dentre os alunos participantes de programas educativos que incluem especialmente a promoção de habilidades sociais, nove por cento reduziram a frequência de problemas de comportamento como a agressividade, $10 \%$ diminuíram sinais de ansiedade e depressão, $23 \%$ melhoraram suas habilidades sociais e $11 \%$ melhoraram 0 desempenho em testes acadêmicos. Resultados como esses apontam para a importância e a necessidade de programas de habilidades sociais para escolares e de sua inclusão regular no currículo das escolas.

A proposição de programas de habilidades sociais na escola foi também destacada por Gresham (2004) defendendo três vertentes de diferentes amplitudes, complementares e sequenciadas, na implantação desses programas na escola. Os programas universais, para todas as crianças da escola, os seletivos, para crianças de risco em pequenos grupos que não se beneficiaram dos universais, e os individualizados, para atendimento das crianças que não respondem positivamente às duas estratégias anteriores.

Sob esse enfoque, considera-se que, dentro dos limites do reduzido tamanho da amostra e da delimitação da faixa etária, a compreensão sobre as possíveis relações entre o comportamento social e o desempenho acadêmico de crianças em idade escolar constitui uma importante base para o planejamento de programas tanto os mais abrangentes, como os mais focalizados. Enquanto os problemas de comportamento de crianças com dificuldades de aprendizagem costumam ser o foco principal da atenção de psicólogos e educadores, as evidências produzidas no presente estudo justificam ampliar esse foco para a avaliação das habilidades sociais desses alunos, dada a sua correlação com o desempenho acadêmico.

Essa perspectiva teria, ainda como efeito concomitante provável, e desejável, a redução de problemas de comportamento, uma vez que as habilidades sociais são equivalentes funcionais, em termos de comportamentos substitutivos, dos problemas de comportamento (GRESHAM, 2009). Em outras palavras, o investimento em habilidades sociais na escola pode ter efeito direto e indireto, tanto sobre o desempenho acadêmico, como sobre os problemas que interferem nesse desempenho, contribuindo para estabelecer uma rede de interdependências positivas entre variáveis determinantes do sucesso escolar e do desenvolvimento da criança.

A avaliação multimodal dos comportamentos sociais aqui empreendida, com pais e professores como avaliadores distintos das 
habilidades sociais de crianças, reforçou a confiabilidade dos dados concordantes. Novos estudos são necessários, com maior número de participantes, verificando a associação dessas variáveis em grupos diferenciados pela presença e ausência de dificuldades de desempenho acadêmico e de comportamento. Os dados obtidos, dentro dos limites do estudo, podem favorecer o planejamento de programas de promoção de habilidades sociais no contexto escolar e de intervenção com crianças com dificuldades de aprendizagem.

\section{Referências Bibliográficas}

BANDEIRA, M.; DEL PRETTE, Z. A. P.; DEL PRETTE, A.; MAGALHÃES, T. Validação das escalas de habilidades sociais, Comportamentos problemáticos e competência acadêmica (SSRS-BR) no ensino fundamental. Psicologia: Teoria e Pesquisa, Brasília, v. 25, n. 2, p. 271-282, 2009.

BANDEIRA, M.; ROCHA, S. S.; SOUZA, T. M. P.; DEL PRETTE, Z. A. P.; DEL PRETTE, A. Comportamentos problemáticos em estudantes do ensino fundamental: Características da ocorrência e relação com habilidades sociais e dificuldades de aprendizagem. Estudos de Psicologia, Natal, v. 11, n. 2, p. 199-208, 2006.

CAPRARA, G. V.; BARBARANELLI, C.; PASTORELLI, C.; BANDURA, A.; ZIMBARDO, P. G. Prosocial foundations of children's academic achievement. Psychological Science, Washington, v. 11, n. 4, p. 302-306, 2000.

CARLSON, C. I. Social interaction goals and strategies of children with learning disabilities. Journal of Learning Disabilities, Austin, v. 20, n. 5, p. 306-311, 1987.

Casel Collaborative for Academic, Social, and Emotional Learning. Social and emotional learning (SEL) and student benefits: Implications for the Safe Schools/Healthy Students Core Elements, Illinois, 2008. Disponível em: <http://www.casel.org/>. Acesso em: 21 Jul. 2009.

DEL PRETTE, A.; DEL PRETTE, Z. A. P. Psicologia das relações interpessoais: Vivências para o trabalho em grupo. Petrópolis: Vozes, 2001.

- Aprendizagem socioemocional na infância e prevenção da violência: Questões conceituais e metodologia da intervenção. In: (Orgs.). Habilidades sociais, desenvolvimento e aprendizagem: Questões conceituais, avaliação e intervenção. Campinas: Alínea, 2003, p. 83-127.

DEL PRETTE, Z. A. P. Prevenção de dificuldades de aprendizagem e da violência: Desenvolvendo tecnologia para avaliação e promoção de habilidades. Relatório de auxílio à pesquisa no exterior (pós-doutorado) aprovado pela FAPESP, Processo número 02-00105-2. São Paulo, SP, Brasil, 2003. 
DEL PRETTE, Z. A. P.; DEL PRETTE, A. Psicologia das habilidades sociais na infância: Teoria e Prática. Petrópolis: Vozes, 2005.

DEL PRETTE, Z. A. P.; DEL PRETTE, A.; OLIVEIRA, L. A.; GRESHAM, F. M.; VANCE, M. J. Role of social performance in predicting learning problems: Prediction of risk using logistic regression analysis, s.d. Submetido.

DIPERNA, J. C. Academic enablers and student achievement: implications for assessment and intervention services in the schools. Psychology in the Schools, Hoboken, v. 43, n. 1, p. 7-17, 2006.

EISENHOWER, A. S.; BAKER, B. L.; BLACHER, J. Early studentteacher relationships of children with and without intellectual disability: Contributions of behavioral, social, and self-regulatory competence. J ournal of School Psychology, New York, v. 45, n. 4, p. 363-383, 2007.

FAD, K. S. The fast track to success: Social-behavioral skills. Intervention in School and Clinic, Austin, v. 26, n. 1, p. 39-43, September 1990.

FEITOSA, F. B. Habilidades sociais e desempenho acadêmico: Processos cognitivos como moderadores e mediadores. Tese de doutorado não publicada. Programa de Pós-Graduação em Educação Especial, Universidade Federal de São Carlos-UFSCar, São Carlos, 2007.

GIL, A. C. Métodos e técnicas de pesquisa social. 5.ed. São Paulo: Atlas. 1999.

GRESHAM, F. M. Current status and future directions of school-based behavioral interventions. School Psychology Review, Bethesda, v. 33, n. 3, p. 326-343, 2004.

GRESHAM, F. M.; ELLIOTT, S. N. Social Skills Rating System: Manual. USA: American Guidance Service, Inc., 1990.

Gresham, F. M. Análise do comportamento aplicada às habilidades sociais. In: DEL PRETTE, A.; DEL PRETTE, Z. A. P. (Orgs.). Psicologia das habilidades sociais: Diversidade teórica e suas implicações. Petrópolis: Vozes, 2009, p. 17-66.

IBOPE/ABEP (2003). Critério de Classificação Econômica Brasil. Diponível em: <http://www.abep.org>. Acesso em: 11 Nov. 2004. JOHNSON, D. W.; JOHNSON, R. T. The Three Cs of promoting social and emotional learning: cooperation, conflict resolution, and civic values. The CEI C Review, Philadelphia, v. 10, n. 6, p. 10-11, 2001.

KAVALE, K. A.; FORNESS, S. R. Social skills deficits and learning disabilities: A meta-analysis. Journal of Learning Disabilities, Austin, v. 29, n. 3, p. 226-237, 1996.

KAZDIN, A. E.; WEISZ, J. R. Context and background of evidencebased psychotherapies for children and adolescents. In (Orgs.). Evidence-Based psychotherapies for children and adolescent. New York: The Guilford Press, 2004, p. 3-20. 
MARTURANO, E. M.; PARREIRA, V. L. C.; BENZONI, S. A. G. Crianças com queixa de dificuldade escolar: Avaliação das mães através da Escala Comportamental Infantil A2 de Rutter. Estudos de Psicologia, Campinas, v. 14, n. 3, p. 3-15, 1997.

MCCLELLAND, M. M.; ACOCK, A. C.; MORRISON, F. J. The impact of kindergarten learning-related skills on academic trajectories at the end of elementary school. Early Childhood Research Quarterly, New York, v. 21, n. 4, p. 471-490, 2006.

MOLINA, R. C. M.; DEL PRETTE, Z. A. P. Funcionalidade da relação entre habilidades sociais e dificuldades de aprendizagem. Psico-USF, Bragança Paulista, v. 11, n. 1, p. 53-63, 2006.

NOVAK, G.; PELÁEZ, M. Child and adolescent development: a behavioral systems approach. London: SAGE Publications, 2003.

Rimm-Kaufman, S. E.; Chiu, Y. Promoting social and academic competence in the classroom: A intervention study examining the contribution of the "Responsive Classroom" approach. Psychology in the Schools, Hoboken, v. 44, n. 4, p. 397-413, 2007.

ROMARO, R. A.; CAPITÃO, C. G. Caracterização da clientela da clínica-escola de psicologia da Universidade de São Francisco. Psicologia: Teoria e Prática, São Paulo, v. 5, n. 1, p. 111-121, 2003.

RUTHERFORD, L. E.; DUPAUL, G. J.; JITENDRA, A. K. Examining the relationship between treatment outcomes for academic achievement and social skills in school-age children with attention-deficit hyperactivity disorder. Psychology in the Schools, Hoboken, v. 45, n. 2, p. 145-157, 2008.

SPSS for Windows. Base system, release 14.0. Chicago, SPSS Inc., 2005.

STEIN, L. M. Teste de Desempenho Escolar (TDE). São Paulo: Casa do Psicólogo, 1994.

SWANSON, H. L.; MALONE, S. Social skills and learning disabilities: A meta-analysis of the literature. School Psychology Review, Bethesda, v. 21, n. 3, p. 427-443, 1992.

WENTZEL, K. R. Relations between social competence and academic achievement in early adolescence. Child Development, Hoboken, v. 62, n. 5, p. 1066-1078, October 1991.

ZINS, J.; WALBERG, H.; WEISSBERG, R. Getting to the heart of the school reform: Social and emotional learning for academic success. National Association of School Psychologists. Communique Bethesda, v. 33, n. 5, p. 35, 2004.

\section{Endereço para correspondência}

Fabio Biasotto Feitosa

Universidade Federal de Rondônia, Departamento de Psicologia, Câmpus Rodovia BR 364, Km 9,5, CEP 76800-000, Porto Velho - RO, Brasil

Endereço eletrônico: fabiobfeitosa@yahoo.com.br

Zilda A. P. Del Prette 
Fabio Biasotto Feitosa, Zilda A. P. Del Prette, Almir Del Prette, Sonia Regina

Universidade Federal de São Carlos, Departamento de Psicologia, Via Washington Luiz, km 235; CEP 13565-905, CP 676, São Carlos - SP, Brasil

Endereço eletrônico: zdprette@ufscar.br

Almir Del Prette

Universidade Federal de São Carlos, Departamento de Psicologia, Via Washington Luiz, km 235; CEP 13565-905, CP 676, São Carlos - SP, Brasil

Endereço eletrônico: adprette@ufscar.br

Sonia Regina Loureiro

R. Tenente Catão Roxo, 2650, CEP 14051-140, Ribeirão Preto-SP , Brasil

Endereço eletrônico: srlourei@fmrp.usp.br

Recebido em: 19/07/2010

Reformulado em: 26/10/2010

Aceito para publicação em: 28/10/2010

Acompanhamento do processo editorial: Adriana Benevides Soares

\section{Notas}

* Psicólogo, Professor Doutor do Departamento de Psicologia da Universidade Federal de Rondônia-UNIR, Porto Velho, Brasil, coordenador do Laboratório de Relações Interpessoais e Saúde, LARIS (www.laris.unir.br).

** Psicóloga, Professora Doutora do Departamento de Psicologia da Universidade Federal de São Carlos, São Carlos, Brasil, Pesquisadora Nível 1 do CNPq, coordenadora do Grupo de Pesquisa Relações Interpessoais e Habilidades Sociais, RIHS (www.rihs.ufscar.br).

*** Psicólogo, Doutor em Ciências, Pesquisador Nível 1 do CNPq.

**** Psicóloga, Professora Doutora do Departamento de Neurociências e Ciências do Comportamento da Faculdade de Medicina de Ribeirão Preto-USP, São Paulo, Brasil, Pesquisadora do CNPq.

${ }^{1}$ Artigo elaborado a partir da tese de doutorado do primeiro autor com o apoio da CAPES e com análises complementares não contidas no trabalho original.

${ }^{2}$ Este instrumento (IBOPE/ABEP, 2003, http://www.abep.org) avalia o poder de compra dos respondentes baseado na posse de bens de consumo, nível educacional do chefe da família e outros fatores, dividindo a população brasileira em cinco classes, que, no ano 2000 , era composta como segue: A1 (1\%), A2 (5\%), B1 $(9 \%), B 2(14 \%), C(36 \%), D(31 \%)$ and $E(4 \%)$. 